\title{
Effect of Carbon Addition on Magnetic Order in Mn-Al-C Alloys
}

\author{
Muriel Tyrman ${ }^{1,2}$, Alexandre Pasko ${ }^{1}$, Loïc Perrière ${ }^{3}$, Victor Etgens ${ }^{2}$, Olivier Isnard ${ }^{4}$ and Frédéric Mazaleyrat ${ }^{1}$ \\ ${ }^{1}$ SATIE UMR 8029 CNRS, ENS Paris-Saclay, Cachan, 94235 France \\ ${ }^{2}$ LISV, UVSQ, Vélizy, 78140 France \\ ${ }^{3}$ ICMPE, CNRS/UPEC, Thiais, 94320 France \\ ${ }^{4}$ Institut Néel, CNRS/UGA, Grenoble, 38042 France
}

\begin{abstract}
Obtaining $100 \%$ of metastable $\tau$-phase $\left(\mathrm{L1}_{0}\right)$ in Mn-Al alloys needs addition of carbon and Mn in excess to stabilize the phase. The excess of Mn could lead to partial antiferromagnetic coupling, that would result in a reduction in magnetization, which is in agreement with the experimental results. To clarify this question, $\left(\mathrm{Mn}_{0.55} \mathrm{Al}_{0.45}\right)_{100-\mathrm{x}} \mathrm{C}_{\mathrm{x}}$ alloys, with $\mathbf{x}$ between $\mathbf{0}$ and 2 , were rapidly quenched from the melt (by melt-spinning) and annealed at $550{ }^{\circ} \mathrm{C}$. In the as-cast state, the sample is in the hexagonal paramagnetic $\varepsilon$-phase, and after annealing, the sample is in the tetragonal ferromagnetic $\tau$-phase. Different routes for the addition of carbon were used. The structural properties were determined by neutron diffraction, and the magnetic properties by means of VSM measurements and neutron diffraction (ND).
\end{abstract}

Index Terms-Neutron diffraction, Magnetic moment, Magnetic coupling, Weiss plot, Structural properties

\section{INTRODUCTION}

A T the scale of the global economy, rare-earth elements are strategic and polluting metals, on which only one country has the monopoly over their refining. For those reasons, it is important to develop rare-earth free magnets with similar properties as rare-earth magnets in order to ensure our economic independence, and to reduce the chemical and radioactive waste associated with their refining. Actually, it is not necessary to have magnets as powerful as the Nd-Fe$B$ magnets, especially in the case of electrical machines for automotive drives [1]. Indeed, the use of the double excitation (magnets and coils) and the modification of the design of the machines make possible to reduce the quantity of magnets at a constant power density.

The Mn-Al alloys whose potential has recently been shown does not present economic and ecological risks. The manganese $(\mathrm{Mn})$ is an interesting element for the creation of new magnets, because this metallic element is abundant and not expensive and carry a strong magnetic moment. However, Mn atoms tend to couple antiferromagnetic, which cancels the magnetization. $\mathrm{Mn}$ atoms couple ferromagnetic when associated with diamagnetic and/or paramagnetic elements. This was first observed in Heusler alloys and later in equiatomic alloys such as hexagonal MnBi or tetragonal $(\tau)$ MnAl. Though the $\mathrm{Mn}$ are only half of the atoms, the magnetization remains relatively high, over $100 \mathrm{Am}^{2} / \mathrm{kg}$.

Since the hard magnetic $\tau$-phase $\left(\mathrm{L1}_{0}\right)$ of $\mathrm{Mn}-\mathrm{Al}$ is metastable, the melt-spinning route is suitable to form this ferromagnetic phase [2,3]. This synthesis technique allows to freeze the high temperature paramagnetic $\varepsilon$-phase, which transform into the ferromagnetic $\tau$-phase after annealing at around $550 \mathrm{C}$. However, formation of $\tau$-phase requires an excess in manganese which can lead to partial antiferromagnetic coupling, i.e. ferrimagnetism. The addition of carbon stabilizes both phases but this addition can have an effect on the magnetic coupling through the deformation of the crystal lattice $[4,5]$.
In principle, the magnetic moment of a ferromagnetic material can be determined by two methods: by measuring the saturation magnetization in the ferromagnetic state at low temperature, or by measuring the high temperature susceptibility. A Weiss plot (reciprocal susceptibility $1 / \chi$ versus temperature) allows to determine the Curie constant and the Curie temperature, and consequently the magnetic moment per $\mathrm{Mn}$ atom in the paramagnetic state of $\tau$-phase. In principle, according to Rhodes and Wohlfarth, in equiatomic composition of Mn- $M$ alloys (with $M$ a metallic atom), the ratio between the paramagnetic moment and the ferromagnetic moment is 1 as the magnetism is expected to be localised electron type because of the half-filled 3d-layer. However, in our case, manganese is in excess and the moment in the paramagnetic state is in all alloys higher than the moment in the ferromagnetic state, which is a characteristic of itinerant electron magnetism. The results suggest that the ferromagnetism of the Mn-Al alloys is not perfect, there is either a non-collinear ferromagnetic coupling or ferrimagnetic coupling.

In order to verify these hypothesis, powder neutron diffraction (ND) has been performed in a wide range of temperatures. The three routes of carbon addition give different results, depending on the quantity of carbon and the homogeneity of the sample. The volume of the unit cell is higher when we use manganese carbide probably because the carbon is well inserted in the $\tau$ lattice. From the ND results, we can determine the average moment per $\mathrm{Mn}$ atom in both ferromagnetic (vector average) and paramagnetic (scalar average because of the localised electron magnetism mechanism) state of $\tau$-phase. The ND result are then compared to the magnetic results.

\section{EXPERIMENTAL Procedure}

A straightforward way to obtain the tetragonal $\mathrm{L}_{0}$ ferromagnetic $\tau$-phase is first freezing the hexagonal $\varepsilon$-phase. To this end, the $\varepsilon$-phase is synthesized by met-spinning. The melt-spun ribbons are then annealed at $550 \mathrm{C}$ in a tubular furnace under argon. Three routes were used for the addition 
of carbon in $\left(\mathrm{Mn}_{0.55} \mathrm{Al}_{0.45}\right)_{100-\mathrm{x}} \mathrm{C}_{\mathrm{x}}$ alloys : (1) addition of graphite pieces in the melt of manganese and aluminium, (2) addition of graphite powder in aluminium tubes and the tubes were melted with the manganese, (3) melting manganese carbide with manganese and aluminium. Melting was realized by induction in cold crucible, the quantity of carbon introduced at this stage is 2 at.\%. The final quantity of carbon in the samples was determined by combustion method by Bureau Veritas. For the samples named Synt 1 et 3 , the final quantity of carbon is $1.88 \%$ and the quantity is $0.98 \%$ for Synt 2 .

For the characterisation, all samples were ground in a mortar. The ND measurements were performed at the ILL (Grenoble, France) on the D1B experiment. The ND were realized in $\theta-2 \theta$ geometry in the angular range $0<2 \theta<130$ with a step of 0,01 at $\lambda=2,52 \AA$. The annealed samples where poured in a vanadium sample holder placed into a cryostat allowing measurements between 2 and $300 \mathrm{~K}$.

The magnetic properties were determined by means of a vibrating sample magnetometer (Lake-Shore VSM). The measurements at high temperatures were carried out under argon flow from room temperature ( $294 \mathrm{~K}$ ) to $523 \mathrm{~K}$ with a step of $50 \mathrm{~K}$. Temperature cycles where performed on the samples in the $\varepsilon$-phase from $294 \mathrm{~K}$ to $900 \mathrm{~K}$ and then from $900 \mathrm{~K}$ down to $294 \mathrm{~K}$. High field magnetic measurements were carried out on all the samples in $\tau$-phase at the INSP (Paris institute of nanosciences, Université Pierre et Marie Curie) up to $9 \mathrm{~T}$ at $20 \mathrm{~K}$ and $300 \mathrm{~K}$ (Quantum Design, PPMS-VSM).

\section{RESULTS AND DISCUSSION}

\section{A. Structural caracterisation}

Rietveld refinement was carried out on all ND diffractrograms using FullProf software (Fig. 1). For all samples, the background noise is much less than conventional XRD, so it is easier to testify the absence of non-magnetic phases $\left(\beta, \gamma_{2}\right)$. For all samples, the results shows single $\tau$-phase.

Regarding the lattice parameters, the results are presented in Fig.2. The parameters $a$ and $c$ increase with temperature. The ratio between $c$ and $a$ doesn't depend on the temperature, that means that the increase of both parameters is due to thermal expansion. The values obtained for the $c$ parameter are the same for Synt 1 and 3, and are higher than for Synt 2. Since the quantity of carbon is $1.88 \%$ for Synt 1 and 3, and only $0.98 \%$ for Synt2, the ND results confirm the influence of the carbon on the lattice parameter, especially on the $c$ parameter. The graph of the ratio $c / a$ shows the deformation in the $c$ direction due to the addition of carbon. At room temperature, the volume of the unit cell for Synt 1,2 and 3 are respectively of $27.72,27.66$ and $27.77 \AA^{3}$. Since without carbon the theoretical volume of the unit cell is $26.19 \AA$, it is clear that carbon increases the volume of the lattice, as an insertion element, of about $6 \%$ for Synt 3 for example. The density versus temperature curves show a decrease of the density with the temperature, which correspond to the increase of the unit cell volume. Synt 3 present the higher unit cell volume, and the smaller density when comparing the three samples.

ND was first carried out on $\varepsilon$-phase. The result presented on Fig. 3 shows two diffraction halos which in principle are

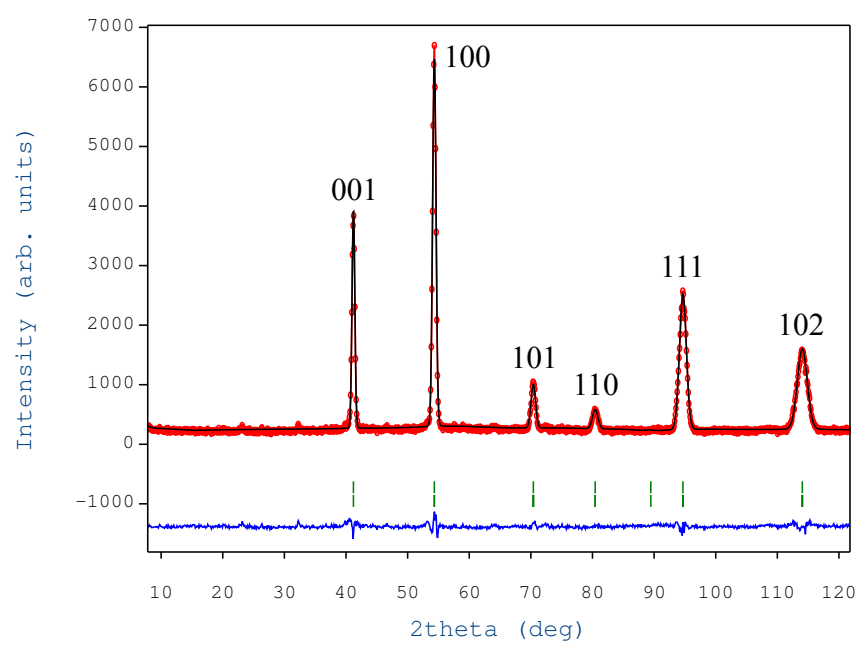

Fig. 1. FullProf refinement for Synt 1 at $10 \mathrm{~K}$.

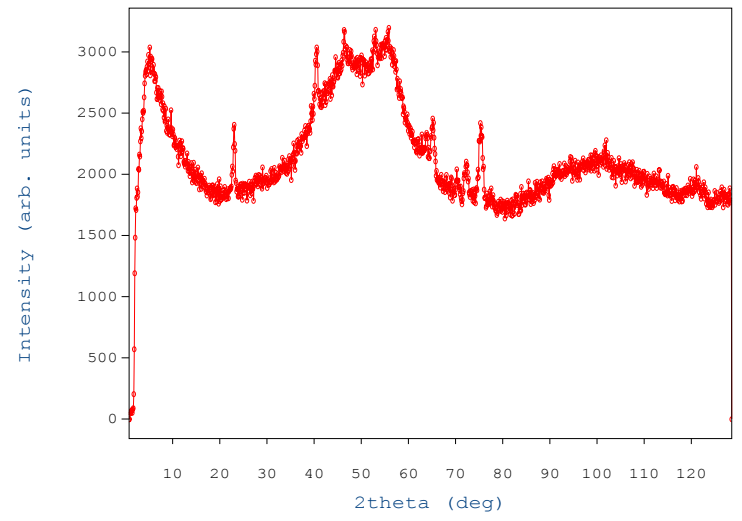

Fig. 3. Diffractogram of $\varepsilon$-phase for Synt 3.

typical of amorphous materials, whereas XRD gives regular patterns of the phase. Indeed, the coherent diffusion lengths are $\bar{b}=3.449 \times 10^{-15} \mathrm{~m}$ for $\mathrm{Al}, \bar{b}=-3.730 \times 10^{-15} \mathrm{~m}$ for $\mathrm{Mn}$ and $\bar{b}=6.646 .10^{-15} \mathrm{~m}$ for $\mathrm{C}$ considering the interaction with thermal neutrons. Since the atomic chemical formula is $\mathrm{Mn}_{54} \mathrm{Al}_{44} \mathrm{C}_{2}$, the coherent diffusion total length is $\bar{b}=-0.3637 \times 10^{-15} \mathrm{~m}$. As a consequence the local disorder of the $\varepsilon$-phase contribution of $\mathrm{Mn}$ and $\mathrm{Al}$ atoms produce destructive interferences. By opposition, due to the layered structure of $\mathrm{L}_{0}$ phase, $\mathrm{Mn}$ and $\mathrm{Al}$ planes diffracts normally.

\section{B. Magnetic structure : Mn magnetic moment.}

In order to determine the average magnetic moment of $\mathrm{Mn}$ atoms, two states of the $\tau$-phase are considered here : the ferromagnetic state for a temperature under $600 \mathrm{~K}$ and the paramagnetic state for a temperature upon $600 \mathrm{~K}$. At high temperature (above the Curie temperature $\left(T_{c}\right)$, the magnetic moment of Mn atoms are randomly oriented. On the contrary, at low temperature, the moments are ordered.

1) Average Mn magnetic moment from ferromagnetic state

From high magnetic field measurements (PPMS), the samples are not saturated even at $9 \mathrm{~T}$, so the saturation magnetization is determined by extrapolation of $20 \mathrm{~K}$ hysteresis loops at infinite field. The magnetization versus $1 /\left(\mu_{0} \mathrm{H}\right)^{2}$ is plotted 

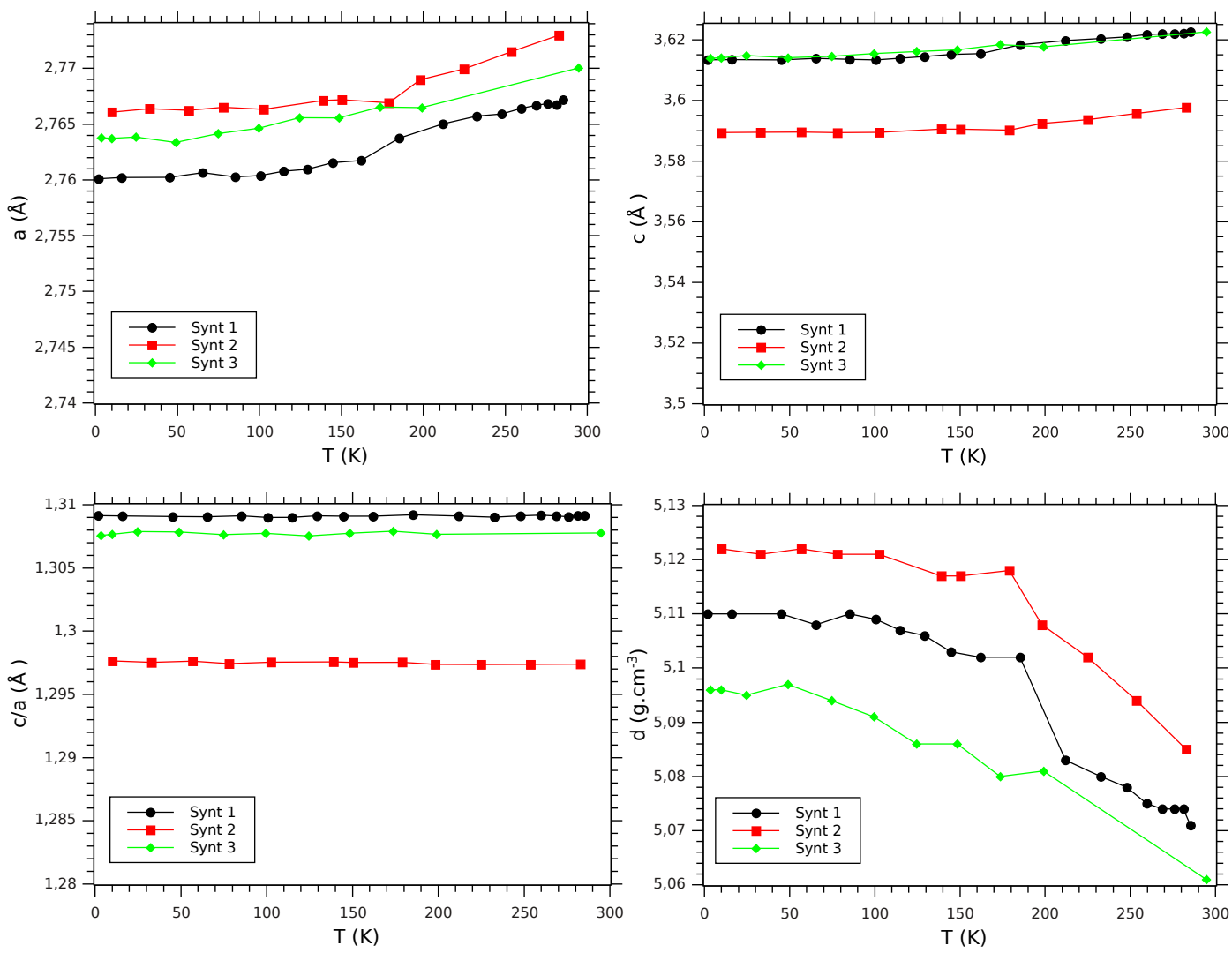

Fig. 2. Lattice parameters and volumic density for the 3 samples versus temperature.

Fig. 4 for Synt 3 for example. The asymptote gives the low temperature spontaneous magnetization $\mathrm{M}_{0}=\mathrm{M}(\infty)$ according to (1):

$$
M(H)=M_{0}+\frac{a}{H^{2}} .
$$

The average magnetic moment, $\left\|\left\langle\overrightarrow{\mathcal{M}_{F}}\right\rangle\right\|=q \mu_{\mathrm{B}}$, is related to the magnetization by :

$$
M_{0}=\frac{\mathcal{N}_{A} \cdot \mathcal{M}}{M_{m o l}}=\frac{\mathcal{N}_{A} q \mu_{B}}{M_{m o l}},
$$

with $\mathrm{n}$ the total number of moles, $\mathcal{N}_{A}$ the Avogadro constant and $\mathrm{M}_{m o l}=41.77836 \mathrm{~g} / \mathrm{mol}$ is the molar mass of $\mathrm{Mn}_{54} \mathrm{Al}_{44} \mathrm{C}_{2}$.

The average number of Bohr magneton per atom is : $\mathrm{q}=0.54 \mathrm{q}_{\mathrm{Mn}}+0.44 \mathrm{q}_{\mathrm{Al}}+0.02 \mathrm{q}_{\mathrm{C}}$. Since only $\mathrm{Mn}$ atoms present a magnetic moment in the samples, $\mathrm{q}_{\mathrm{Al}}=\mathrm{q}_{\mathrm{C}}=0$ here. Finally, in order to determine the average moment per $\mathrm{Mn}$ atom, the equation is : $\left\|\left\langle\overrightarrow{\mathcal{M}_{F}}\right\rangle\right\|=\frac{q}{0.54} \mu_{\mathrm{B}}$.

In the ferromagnetic state of $\tau$-phase, a vectorial average of the magnetic moment is obtained. Considering the magnetic moment per site 1a and 1d (Fig. 5), the calculated average is presented by the equation 3 , with $n_{1 a}$ and $n_{1 d}$ the number of Mn atoms in both sites:

$$
\left\langle\overrightarrow{\mathcal{M}_{F}}\right\rangle=\frac{1}{n_{1 a}+n_{1 d}}\left(n_{1 a} \overrightarrow{\mathcal{M}_{1 a}}+n_{1 d} \overrightarrow{\mathcal{M}_{1 d}}\right)
$$

expressed in Bohr units and simplified for an antiferromagnetic coupling between $1 \mathrm{a}$ and $1 \mathrm{~d}$ sites. The results for the three samples are presented in table I.

The polarisation $J$ and the magnetization $M$ are related by $J=\mu_{0} \rho_{v} M$, with $\rho_{v}$ the density calculated from the lattice parameters.

TABLE I

FERROMAGNETIC STATE AT $20 \mathrm{~K}$

\begin{tabular}{|c||c|c|c|c|}
\hline Synt. & $\begin{array}{c}\left\|\left\langle\overrightarrow{\mathcal{M}_{F}}\right\rangle\right\| \\
\left(\mu_{B}\right)\end{array}$ & $\begin{array}{c}M(\infty) \\
\left(\mathrm{Am}^{2} \mathrm{~kg}^{-1}\right)\end{array}$ & $\begin{array}{c}\mathrm{J}_{0} \\
(\mathrm{~T})\end{array}$ & $\begin{array}{c}\rho_{v} \\
\left(\mathrm{~kg} . \mathrm{m}^{-3}\right)\end{array}$ \\
\hline \hline Synt 1 & 1.871 & 135.08 & 0.867 & 5109 \\
\hline Synt 2 & 1.974 & 142.49 & 0.917 & 5122 \\
\hline Synt 3 & 1.863 & 134.49 & 0.861 & 5096 \\
\hline
\end{tabular}

The addition of carbon decreases the average magnetic moment for the $\tau$-phase in the ferromagnetic state, presumably as a result of band structure modification.

\section{2) Weiss plot}

The Weiss plot for Synt 3 is given as an example on figure 6. The black curve corresponds to the heating of the sample in the $\epsilon$-phase and the drop reveals the $\varepsilon \rightarrow \tau$ transformation. The red curve corresponds to the cooling part after the transformation. All the sample are paramagnetic above $600 \mathrm{~K}$ and the decrease of $1 / \chi$ with the temperature is linear as represented by the blue line. This line gives the paramagnetic $T_{c}$ by the intersection between the line and the abscissa axis, and the Curie constant 


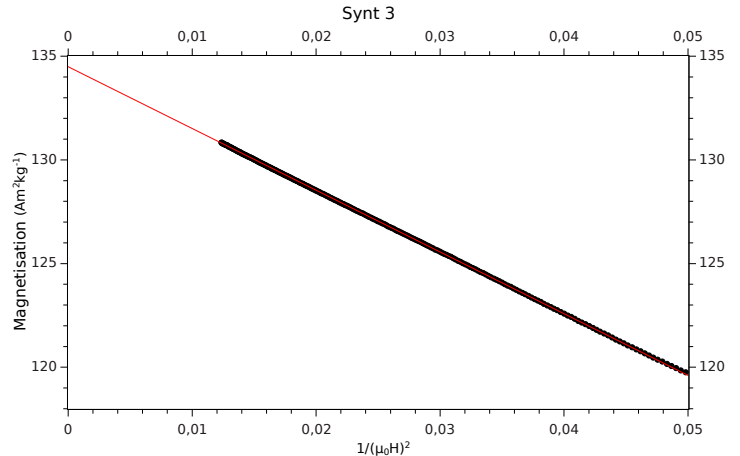

Fig. 4. $\mathrm{M}=\mathrm{f}\left(\left(1 /\left(\mu_{0} \mathrm{H}\right)^{2}\right)\right.$ for Synt 3 at $20 \mathrm{~K}$.

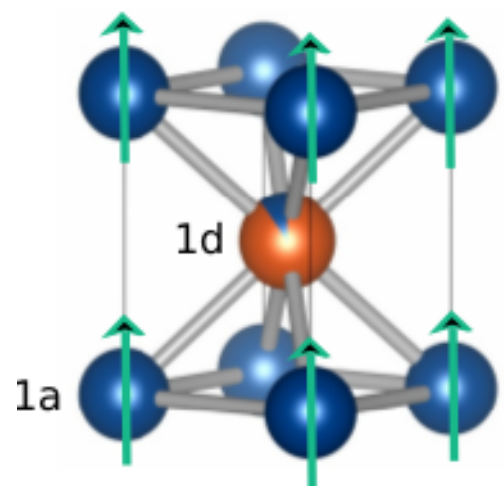

Fig. 5. 1a and 1d sites of $\mathrm{Mn}-\mathrm{Al} \tau$-phase.

$C$ which is related to the slope of this line, in one hand, and to the average moment per atoms in the over hand. Here the measured magnetic moment is a scalar average of atomic moments:

$$
\left\langle\left\|\overrightarrow{\mathcal{M}_{F}}\right\|\right\rangle=\sqrt{\frac{C . k_{B}}{\mu_{0} \cdot N}},
$$

where kis the Boltzmann constant and $\mu_{0}$ the vacuum permeability. The number of particles per unit of volume is :

$$
N=\frac{\rho_{v} \cdot \mathcal{N}_{A}}{M} \approx 7.452 \times 10^{28} \mathrm{~m}^{-3},
$$

with $\rho_{v}=5170 \mathrm{~kg} \cdot \mathrm{m}^{-3}$ the theoretical density for the samples at room temperature.

Afterward, the average moment per $\mathrm{Mn}$ atom is calculated the same way as for the ferromagnetic state.

For the paramagnetic state, the average is scalar, means the equation 7 can be written as a function of $n_{1 a}$ and $n_{1 d}$, the number of $\mathrm{Mn}$ atoms in each site.

$$
\begin{array}{r}
\left\langle\left\|\overrightarrow{\mathcal{M}_{F}}\right\|\right\rangle=\frac{1}{n_{1 a}+n_{1 d}}\left(n_{1 a}\left\|\overrightarrow{\mathcal{M}_{1 a}}\right\|+n_{1 d}\left\|\overrightarrow{\mathcal{M}_{1 d}}\right\|\right) \\
=\frac{n_{1 a} q_{1 a}+n_{1 d} q_{1 d}}{n_{1 a}+n_{1 d}}
\end{array}
$$

The results presented in table II show that the average moment in the paramagnetic state is much higher than the one
TABLE II

MAGNETIC PARAMETERS FROM WEISS PLOTS AND FERROMAGNETIC RESULTS.

\begin{tabular}{|c||c|c|c|c|}
\hline Synt. & $\mathrm{T}_{\text {trans }}$ & $\begin{array}{c}\mathrm{T}_{\text {Curie }} \\
(\mathrm{K})\end{array}$ & $\begin{array}{c}\left\|\left\langle\overrightarrow{\mathcal{M}_{F}}\right\rangle\right\| \\
\text { ferro } \\
\left(* \mu_{B}\right) \text { at } 20 \mathrm{~K}\end{array}$ & $\begin{array}{c}\left\langle\left\|\overrightarrow{\mathcal{M}_{F}}\right\|\right\rangle \\
\text { para } \\
\left(* \mu_{B}\right) \mathrm{T}>600 \mathrm{~K}\end{array}$ \\
\hline \hline Synt 1 & 760 & 580 & 1.871 & 2.88 \\
\hline Synt 2 & 781 & 598 & 1.974 & 2.91 \\
\hline Synt 3 & 780 & 582 & 1.863 & 3.01 \\
\hline
\end{tabular}

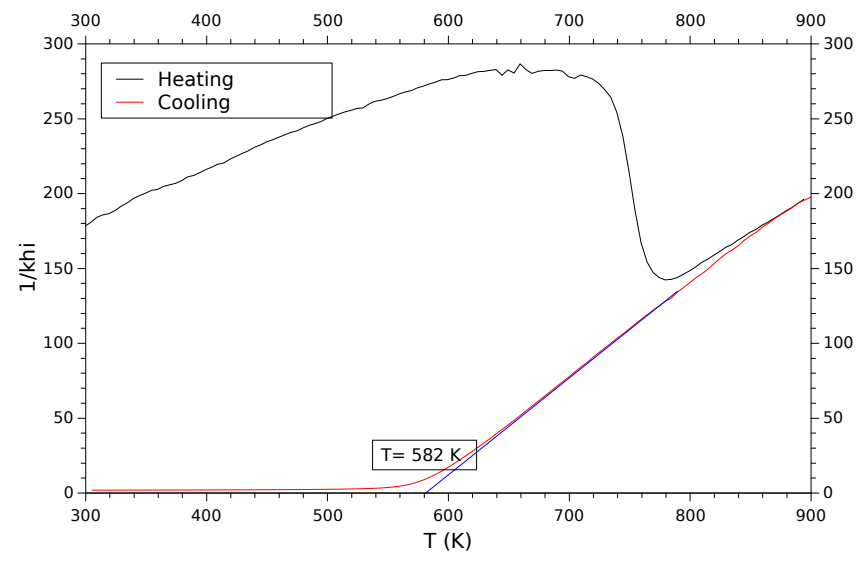

Fig. 6. Weiss plot of Synt $3,1 / \chi=\mathrm{f}(\mathrm{T})$.

in the ferromagnetic state which points out the non collinear nature of the ferromagnetism of these samples.

\section{Neutron diffraction}

The advantage of ND technique is its sensitivity to magnetic moment magnitude and direction. With the FullProf software on ND diffractograms, it is possible to refine simultaneously the lattice parameters and the magnetic moments of the Mn atoms [6]. All 1a sites are filled by $\mathrm{Mn}$ atoms and $1 \mathrm{~d}$ sites are filled with the excess of $\mathrm{Mn}$, and with all $\mathrm{Al}$ atoms. The magnetism of both sites was first refined simultaneously. For all the diffractograms of the samples, the magnetic moment of the 1d site present an opposite sign of the moment of the 1a site which is a clear evidence of the ferrimagnetic nature of the samples. For Synt 2, measurements were carried out from $10 \mathrm{~K}$ to $531 \mathrm{~K}$, the higher temperature the cryostat can reach. The figure 7 shows a decrease with the increase of temperature for all the peaks except for the plan (001). Magnetic moments are then oriented to the [001] axis, that is to say the c-axis is the easy axis of magnetization.

The variations with the temperature of the moment carried by the $1 \mathrm{~d}$ site are almost non-existent, and for this reason, the value of the $1 \mathrm{~d}$ site was fixed at $\overrightarrow{\mathcal{M}_{1 d}}=-2,95 \mu_{B} \overrightarrow{u_{c}}$ in all the refinements. This choice comes from a strong uncertainty on this site because only $5 \%_{a t}$ of the atoms are on the $1 \mathrm{~d}$ site and are Mn atoms.

The figure 8 shows the evolution of the 1a site magnetic moment versus temperature for the 3 samples. The scale of the figure shows that the magnetic moment carried by Mn atoms on the 1a sites decrease significantly with the increase of the 


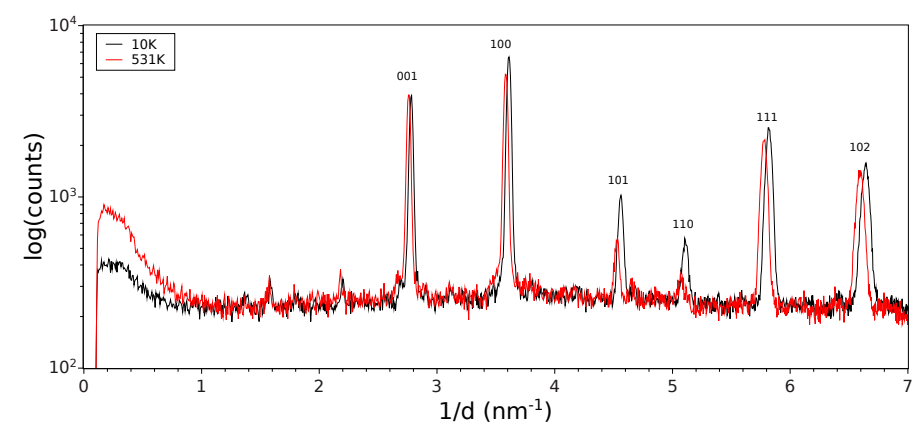

Fig. 7. $1 / \mathrm{d}\left(\mathrm{nm}^{-1}\right)$ diffractogram of Synt 2 at $10 \mathrm{~K}$ and $531 \mathrm{~K}$.

temperature, while for the magnetic measurements, we assume the value of the moment doesn't depend on temperature. This decrease of the magnetic moment can be related to the increase of the volume of the crystallographic lattice with the temperature, corresponding to a widening of the $3 \mathrm{~d}$ magnetic band.

The ND results highlight also a higher 1a site magnetic moment for Synt 3, synthesised from manganese carbide. The unit cell volume of this sample is higher than that of the other samples. As the magnetic moment of 1d site is significantly higher than the moment of 1a site, the increase of the distance between both sites decreases the antiferromagnetic coupling. The electrons are then more localised on the Mn atoms, which also explains the increase of the magnetic moment of the $\mathrm{Mn}$ on the 1a sites of Synt 3. Then the vector average of the magnetization can be calculated from (3) considering antiferromagnetic coupling between $1 \mathrm{a}$ and $1 \mathrm{~d}$ sites:

$$
\left\langle\overrightarrow{\mathcal{M}_{F}}\right\rangle=\frac{n_{1 a} q_{1 a}-n_{1 d} q_{1 d}}{n_{1 a}+n_{1 d}} \overrightarrow{u_{c}},
$$

with $\overrightarrow{u_{c}}$ the unitary vector in the c direction.

When comparing the ND and the magnetometry measurements (Table II), it is seen that the values are of the same order of magnitude and shows the antiferromagnetic coupling between 1a and 1d sites, while the 1a sites are ferromagnetically coupled.

TABLE III

AVERAGE MAGNETIC MOMENT PER MN ATOM IN $\mu_{B}$ UNITS, DETERMINED FROM MAGNETIC MEASUREMENTS AND FROM ND.

\begin{tabular}{|c||c|c||c|c|}
\hline Synt & $\begin{array}{c}\left\|\left\langle\overrightarrow{\mathcal{M}_{F}}\right\rangle\right\| \\
\text { ferro } \\
\text { VSM }\end{array}$ & $\begin{array}{c}\left\langle\left\|\overrightarrow{\mathcal{M}_{F}}\right\|\right\rangle \\
\text { para } \\
\text { VSM }\end{array}$ & $\begin{array}{c}\left\|\left\langle\overrightarrow{\mathcal{M}_{F}}\right\rangle\right\| \\
\text { ferro } \\
\text { ND }\end{array}$ & $\begin{array}{c}\left\langle\left\|\overrightarrow{\mathcal{M}_{F}}\right\|\right\rangle \\
\text { para } \\
\text { ND }\end{array}$ \\
\hline \hline Synt 1 & 1.87 & 2.88 & 1.94 & 2.67 \\
\hline Synt 2 & 1.97 & 2.91 & 1.81 & 2.53 \\
\hline Synt 3 & 1.86 & 3.01 & 2.20 & 2.93 \\
\hline
\end{tabular}

\section{CONCLUSION}

In summary, three ways to introduce carbon in $\mathrm{Mn}-\mathrm{Al}$ were used. The carbide way allows full insertion of $\mathrm{C}$ in the lattice, while the other ways are less efficient from this

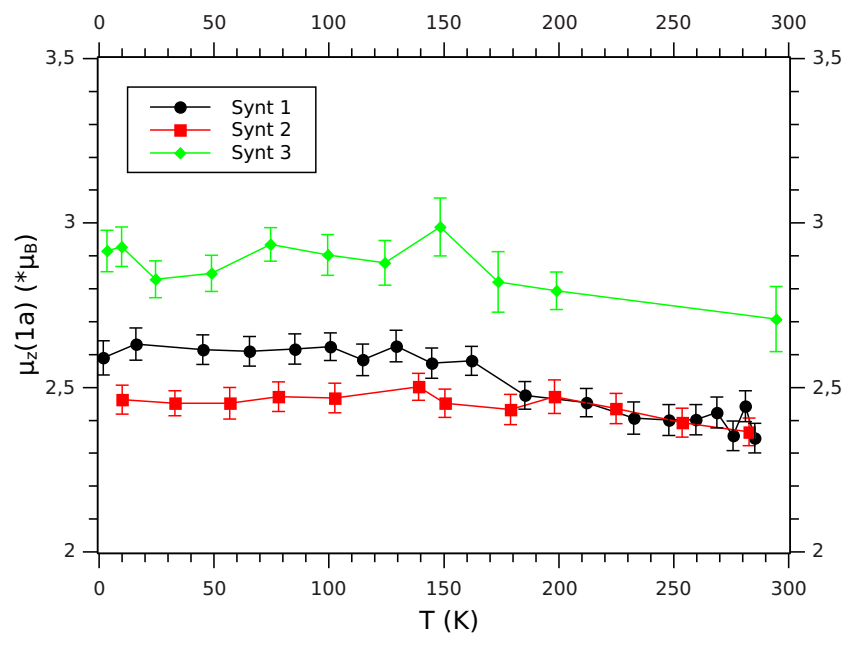

Fig. 8. Magnetic moments of 1a site of $\tau$-phase.

point of view. In all cases, the melt-spinning route - liquid $\rightarrow \epsilon \rightarrow \tau$ transformation - yields the pure tetragonal ordered phase. Calculation of the average magnetic moment from low temperature and high temperature (Weiss plot) measurements indicates the non collinear nature of the magnetic order of $\mathrm{Mn}-\mathrm{Al}$. ND diffraction reveals that $\mathrm{Mn}$ in $1 \mathrm{a}$ and $1 \mathrm{~d}$ sites of the $\mathrm{L} 1_{0}$ phase exhibit magnetic moments in opposition and with different magnitudes. In other words, $\mathrm{Mn}-\mathrm{Al}(\mathrm{C})$ containing an excess of $\mathrm{Mn}$ are ferrimagnetic. These results also demonstrate that wide temperature range magnetometry can be used efficiently to determine the magnetic coupling.

\section{ACKNOWLEDGMENT}

This work was financed by the Industrial Chair Matinnov (Valéo-UVSQ).

\section{REFERENCES}

[1] Coey J. M. D. (2014). New permanent magnets; manganese compounds. J. Phys. Condens. Matter, 26064211.

[2] Liu Z. W., Chen C., Zheng Z. G., Tan B. H., and Ramanujan R. V. (2012). Phase transitions and hard magnetic properties for rapidly solidified $\mathrm{MnAl}$ alloys doped with $\mathrm{C}, \mathrm{B}$, and rare earth elements. J. Mater. Sci., 47 2333-2338.

[3] Fazakas E., Varga L. K., and Mazaleyrat F. (2007). Preparation of nanocrystalline $\mathrm{Mn}-\mathrm{Al}-\mathrm{C}$ magnets by melt spinning and subsequent heat treatments. J. Alloys Comp., 434-435 611613.

[4] Pasko A., Mazaleyrat F., Lobue M., Fazakas E. and Varga L. K. (2013). Hard magnetic properties of melt-spun Mn-Al-C alloys. Eur. Phys. J. Web Conf. 4006008.

[5] Wei J. Z., Song Z. G., Yang Y. B., Liu S. Q., Du H. L., Han J. Z., Zhou D., Wang C. S., Yang Y. C.,Franz A. and Többens D. (2014). $\tau$-MnAl with high coercivity and saturation magnetization. AIP Advances, 4127113.

[6] Em V. T., Latergaus I. S., Remeev A. S., ans Lee C. T. (1997). Neutron Diffraction Study of the $\tau$-Phase of CarbonDoped Mn-Al Alloy. Phys. Stat. Sol., 159(2), 323-326. 